\title{
PREDICTIVE MODELING FOR QUALITY ASSURANCE OF EXTRUSION BLOW MOLDING
}

\section{E.V. RAMANA, VONGUR RAMULU \& N. KIRAN KUMAR}

Professor, Department of Mechanical Engineering, VNR Vignana Jyothi Institute of Engineering and Technology, Bachupally, Hyderabad, India

Research Scholar, M. Tech (AMS), Department of Mechanical Engineering, VNR Vignana Jyothi Institute of Engineering and Technology, Bachupally, Hyderabad, India

Associate Professor, Department of Mechanical Engineering, VNR Vignana Jyothi Institute of Engineering and Technology, Bachupally, Hyderabad, India

\begin{abstract}
In Extrusion Blow molding process, the manufacturing of hollow products isdone by using semi-molten tube (parison). During processing of the product, the defects such as underweight of part, warpage, part does not blow and thinning of the weld arises. The deviations in process variables are responsible in causing the defects in products. Predictive models are developed in finding the relationship between process variables and causes of defects using data mining techniques. This paper presents Random Tree, Naive Bayes (Kernel), and Neural Net, Stacking and Vote techniques in Rapid Miner for data mining. These techniques are implemented on Extrusion Blow Molding Process dataset collected during the manufacture of $100 \mathrm{ml}$ made of high-density polyethylene (HDPE) material in building data mining models for the assurance of quality and predictions of product quality.
\end{abstract}

KEYWORDS: Random Tree, Naive Bayes (Kernel), Neural Net, Stacking, Vote, Extrusion Blow Molding

Received: Jun 08, 2020; Accepted: Jun 28, 2020; Published: Aug 21, 2020; Paper Id.: IJMPERDJUN2020791

\section{INTRODUCTION}

Extrusion blow molding is a manufacturing process where thermoplastic resins are used as processing material. The resin is melted in extruder and parison is extruded out as a tube (parison) through die head. Parison is pinched by pinch bars and then air is blown through blow pins into the parison in order to expand it to take the shape of mould cavity. The part is cooled in the mould and ejected out of mould cavity. Process variables that influence the quality of parts are blow pressure,barrel temperatures, extrusion time, extrusion speed, blow time, cut off blade temperature, ejection time, cycle time, cooling time, mould temperature etc. The process variables have to be continuously monitored and controlled to achieve the required quality and minimize rejection rate.

Huang HX et al. [1] have studied the influence of flow rate and temperature of die on the diameter of parison in extrusion blow molding. Huang GQ et al. [2] have worked on a hybrid method comprising of ANN, FEM and GA to determine optimal parison thickness distribution. Huang HX et al. [3] have developed ANN model to predict the dimensions on the parison at any location. Tahboub KK et al. [4] have implemented DoE approach to optimize the extrusion blow molding process. Ramana E.V et al. [5] have applied SVM; KNN and GC\&RT techniques to predict the quality of injection molding process.Ramana E.V et al. [6] have applied SAAN, GCHAID and Association Rules to predict the quality of injection molding process. 
In the present study, Naive Bayes (Kernel), Random Tree, Neural Net, Vote and Stacking techniques in Rapidminer are implemented for predicting quality and in finding root causes of defects (underweight of part, warpage, thinning of the weld, part does not blow) encountered in $100 \mathrm{ml}$ container.

\section{DATASET}

Extrusion Blow Molding process datasetof $100 \mathrm{ml}$ container made of high-density polyethylene (HDPE) comprises of 200 records that includes numerical and nominal data. Process attribute data related to barrel temperatures at five zones; blow pressure, blow time, cut off blade temperature, cooling time, cycle time, extrusion speed, extrusion time, ejection time are selected for the development of predictive models.

\section{NAIVE BAYES (KERNEL)}

Naive Bayes (Kernel) is a probabilistic classifier and it assumes that input attributes are independent and training data requirement for classification is less. Naive Bayes (Kernel) model is based on all data points to arrive an estimate using kernel density estimators (Non- parametric). The training dataset is provided as input to Naive Bayes (Kernel) operator as shown in the process presented in Figure 1. The laplace correction is applied for avoiding zero probabilities. Greedy option is selected as the estimation mode of kernel density. The prediction accuracy of Naive Bayes (Kernel) model is found to be highest when minimum bandwidth and number of kernels is set as 1.0 and 3 respectively after exploring various other values for these parameters.

Naive Bayes (Kernel) operator delivers model through mod (output) port which is transferred to Apply Model operator through its mod (input) port. The test dataset output (out) port is linked to Apply Model operator by means of the unlabelled (unl) port. The test dataset having class labels which are known is provided as input to performance vector in order to appraise the performance of Naive Bayes (Kernel) model for classification.

Confusion matrix generated with class precision, recall and prediction accuracy of Naive Bayes (Kernel) model on test dataset is shown in Table 1.

Table 1 show the Naive Bayes (Kernel) model predicted products belonging to accepted and part does not blow classes as true positives for all cases. Prediction of underweight of part class resulted in 7 cases as true positives and 1 case as false positive, 1 case of false negative (warpage). Prediction of warpage class resulted in 8 cases as true positives and 3 cases as false positives. Prediction of thinning of the weld class resulted in 5 true positive cases, 1 case false positive (underweight of part), and 2 cases as false negatives (warpage).

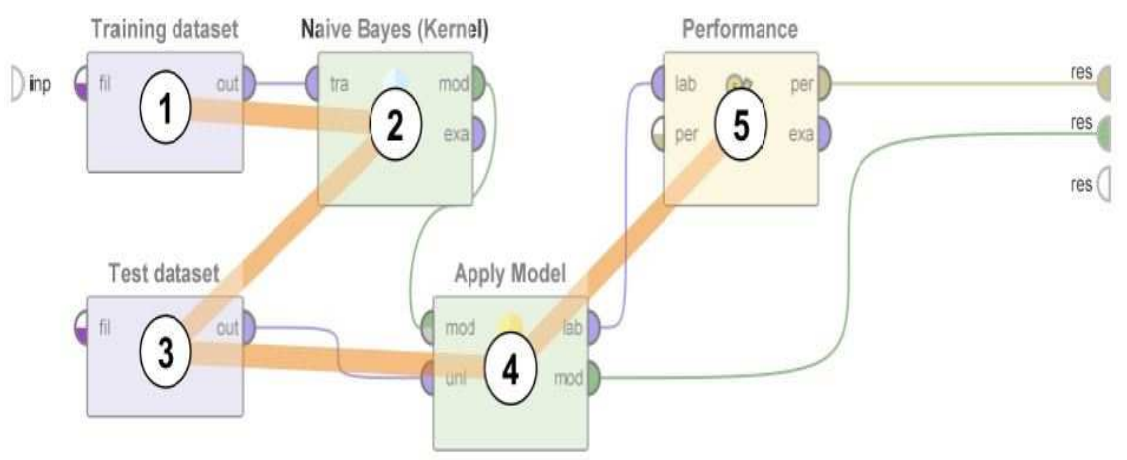

Figure 1: Rapid Miner Process with Naive Bayes (Kernel) Operator. 
Table 1: Confusion Matrix of Naive Bayes (Kernel) Model

\begin{tabular}{|l|c|c|c|c|c|c|}
\hline & $\begin{array}{c}\text { True Under } \\
\text { Weight of Part }\end{array}$ & $\begin{array}{c}\text { True } \\
\text { Warpage }\end{array}$ & $\begin{array}{c}\text { True Thinning } \\
\text { of The Weld }\end{array}$ & $\begin{array}{c}\text { True } \\
\text { Accepted }\end{array}$ & $\begin{array}{c}\text { True Part } \\
\text { Does Not Blow }\end{array}$ & $\begin{array}{c}\text { Class } \\
\text { Precision }\end{array}$ \\
\hline Pred. Underweight of part & 7 & 0 & 1 & 0 & 0 & $87.50 \%$ \\
\hline Pred. Warpage & 1 & 8 & 2 & 0 & 0 & $72.73 \%$ \\
\hline Pred. Thinning of the weld & 0 & 0 & 5 & 0 & 0 & $100.00 \%$ \\
\hline Pred. Accepted & 0 & 0 & 0 & 8 & 0 & $100.00 \%$ \\
\hline Pred. Part does not blow & 0 & 0 & 0 & 0 & 8 & $100.00 \%$ \\
\hline Class recall & $87.50 \%$ & $100.00 \%$ & $62.50 \%$ & $100.00 \%$ & $100.00 \%$ & \\
\hline
\end{tabular}

\section{RANDOM TREE}

Random Tree chooses subset of attributes randomly and involves several input attributes in predicting the class label. The training dataset is provided as input to Random Tree operator as shown in the process presented in Figure 2 . The information gain is selected as a criterion in splitting the attributes. The minimal size for splitting the nodes is and minimal size of the leaf are set as 4 and 5 respectively. The minimal gain before splitting a node is set as 0.1 . Maximum depth is set to 4 for restricting the tree size. Confidence level used in calculation of pessimistic error for pruning the tree is set to value of 0.25 . Pre-pruning alternatives are restricted to a value of 2 in case of splitting is prevented at a certain node while prepruning the tree. The subset ratio parameter is marked for guessing.

Random Tree operator delivers its model through mod (output) port and then transferred to Apply Model operator across mod (input) port. The test dataset output (out) port is linked to Apply Model operator by means of the unlabelled (unl) port. The test dataset having class labels which are known is provided as input to performance vector in order to appraise the performance of Random Tree model for classification.



Figure 2: Rapid Miner Process with Random Tree Operator.

Random Tree built on the training dataset by the model is displayed in Figure 3. The description of Random Tree is presented as given below:

Barrel Temperature Zone $4\left({ }^{\circ} \mathrm{C}\right)>163.500$

| Cycle Time $(\mathrm{sec})>12.250$

| | Barrel Temperature Zone $3\left({ }^{\circ} \mathrm{C}\right)>159.500$ : underweight of part $\{$ underweight of part $=10$, warpage $=0$, thinning of the weld $=0$, accepted $=0$, part does not blow $=0\}$

| | Barrel Temperature Zone $3\left({ }^{\circ} \mathrm{C}\right) \leq 159.500$ : thinning of the weld $\{$ underweight of part $=22$, warpage $=0$, thinning of the weld $=32$, accepted $=0$, part does not blow $=0$ \} 
| Cycle Time $(\mathrm{sec}) \leq 12.250$ : warpage $\{$ underweight of part $=0$, warpage $=32$, thinning of the weld $=0$, accepted $=0$, part does not blow $=0\}$

Barrel Temperature Zone $4\left({ }^{\circ} \mathrm{C}\right) \leq 163.500$

| Cycle Time $(\mathrm{sec})>16$ : part does not blow \{ underweight of part $=0$, warpage $=0$, thinning of the weld $=0$, accepted $=0$, part does not blow $=32\}$

| Cycle Time $(\mathrm{sec}) \leq 16$ : accepted $\{$ underweight of part $=0$, warpage $=0$, thinning of the weld $=0$, accepted $=32$, part does not blow $=0\}$

Confusion matrix generated with class precision, recall and prediction accuracy of Random Tree model on test dataset is shown in Table 2.

The Random Tree model predicted products with warpage, accepted and part does not blow classes as true positives for all cases. Prediction of underweight of part class resulted in 1 case as true positives, 7 cases of false negatives (thinning of the weld). Prediction of thinning of the weld class resulted in 8 cases as true positives and 7 cases as false positives.

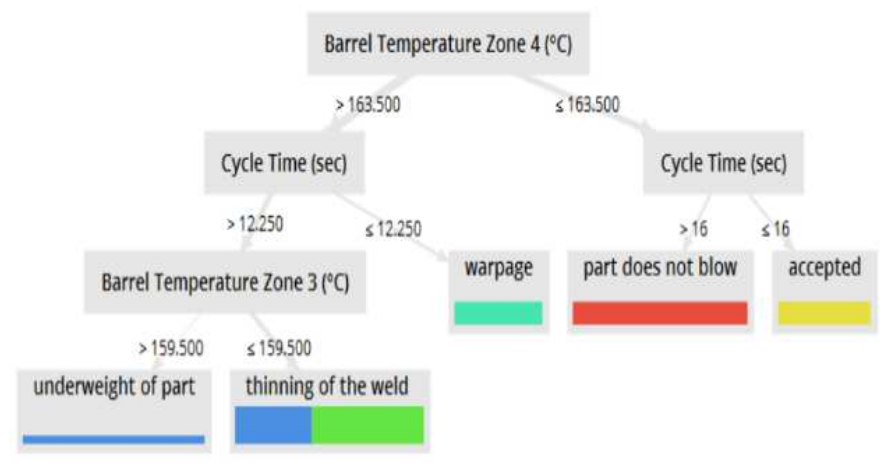

Figure 3: Random Tree.

Table 2: Confusion Matrix of Random Tree Model

\begin{tabular}{|l|c|c|c|c|c|c|}
\hline \multicolumn{7}{|c|}{ Accuracy: 82.50 \% } \\
\hline & $\begin{array}{c}\text { True Under } \\
\text { Weight of Part }\end{array}$ & $\begin{array}{c}\text { True } \\
\text { Warpage }\end{array}$ & $\begin{array}{c}\text { True Thinning } \\
\text { of The Weld }\end{array}$ & $\begin{array}{c}\text { True } \\
\text { Accepted }\end{array}$ & $\begin{array}{c}\text { True Part } \\
\text { Does Not Blow }\end{array}$ & $\begin{array}{c}\text { Class } \\
\text { Precision }\end{array}$ \\
\hline $\begin{array}{l}\text { Pred. Underweight of } \\
\text { part }\end{array}$ & 1 & 0 & 0 & 0 & 0 & $100.00 \%$ \\
\hline Pred. Warpage & 0 & 8 & 0 & 0 & 0 & $100.00 \%$ \\
\hline $\begin{array}{l}\text { Pred. Thinning of the } \\
\text { weld }\end{array}$ & 7 & 0 & 8 & 0 & 0 & $53.33 \%$ \\
\hline Pred. Accepted & 0 & 0 & 0 & 8 & 0 & $100.00 \%$ \\
\hline $\begin{array}{l}\text { Pred. Part does not } \\
\text { blow }\end{array}$ & 0 & 0 & 0 & 0 & 8 & $100.00 \%$ \\
\hline Class recall & $12.50 \%$ & $100.00 \%$ & $100.00 \%$ & $100.00 \%$ & $100.00 \%$ & \\
\hline
\end{tabular}

\section{NEURAL NET}

Neural Net (NN) also called as Artificial Neural Network works emulates biological neural networks in its working. It builds model on feed forward neural network to detect patterns in data. It comprises of single input layer, single or multiple hidden layers and single output layer. Polynomial attributes are not supported by Neural Net operator. The training dataset is provided as input to Neural Net operator as shown in the process presented in Figure 4. Number of training cycles is set to a value of 500 
which resulted in higher prediction accuracy. Learning rate parameter is set to 0.02 to change the weights at every step. The momentum is set to a value of 0.4 that adds a fraction of earlier weight to current one. The parameter shuffle is enabled to shuffle input data before learning. Normalization is applied before learning due to NN operator uses sigmoid function. Error Epsilon parameter is set to $1.0 \mathrm{E}-4$ in order to stop the optimization if the training error falls below this value.

Neural Net operator transfers the model through mod (output) port to Apply Model operator from its mod (input) port. The test dataset is supplied to Apply Model operator by means of the unlabelled (unl) port. The test dataset with class labels is provided as input to performance vector in order to appraise the predictive performance of Neural Net model.

Table 3 presents confusion matrix generated with class precision, recall and prediction accuracy of Neural Net model on test dataset.

The Neural Net model predicted products with warpage, accepted and part does not blow classes as true positives for all cases. Prediction of underweight of part class resulted in 6 cases as true positives, 2 cases as false negatives (thinning of the weld). Prediction of thinning of the weld class resulted in 8 cases as true positives and 2 cases as false positives.

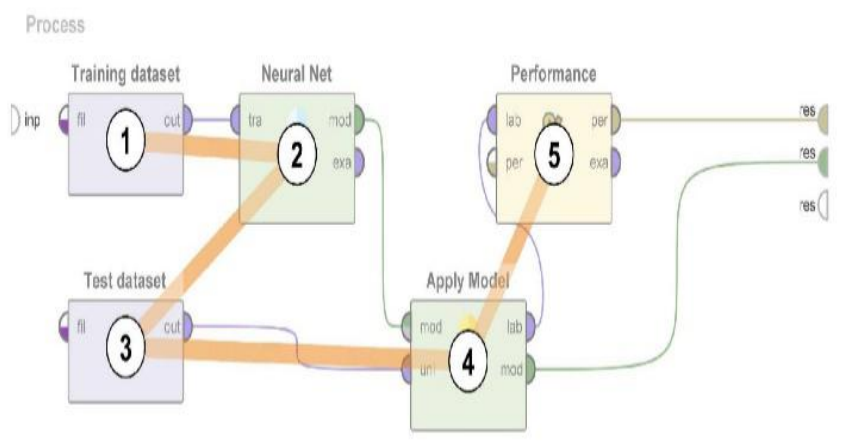

Figure 4: Rapid Miner Process with Neural Net Operator.

Table 3: Confusion Matrix of Neural Net Model

\begin{tabular}{|l|c|c|c|c|c|c|}
\hline & $\begin{array}{c}\text { Accuracy: 95.00 \% } \\
\text { True Under } \\
\text { Weight of Part }\end{array}$ & $\begin{array}{c}\text { True } \\
\text { Warpage }\end{array}$ & $\begin{array}{c}\text { True } \\
\text { Thinning of } \\
\text { The Weld }\end{array}$ & $\begin{array}{c}\text { True } \\
\text { Accepted }\end{array}$ & $\begin{array}{c}\text { True Part Does } \\
\text { Not Blow }\end{array}$ & $\begin{array}{c}\text { Class } \\
\text { Precision }\end{array}$ \\
\hline $\begin{array}{l}\text { Pred. Underweight of } \\
\text { part }\end{array}$ & 6 & 0 & 0 & 0 & 0 & $100.00 \%$ \\
\hline Pred. Warpage & 0 & 8 & 0 & 0 & 0 & $100.00 \%$ \\
\hline $\begin{array}{l}\text { Pred. Thinning of the } \\
\text { weld }\end{array}$ & 2 & 0 & 8 & 0 & 0 & $80.00 \%$ \\
\hline Pred. Accepted & 0 & 0 & 0 & 8 & 0 & $100.00 \%$ \\
\hline Pred. Part does not blow & 0 & 0 & 0 & 0 & 8 & $100.00 \%$ \\
\hline Class recall & $75.00 \%$ & $100.00 \%$ & $100.00 \%$ & $100.00 \%$ & $100.00 \%$ & \\
\hline
\end{tabular}

\section{STACKING}

Stacking is one of the ensemble learning techniques where several models are combined to attain higher performance during prediction in comparison with a single model. The training dataset is provided as input to Stacking operator as shown in the process presented in Figure 5. Stacking operator includes Stacking Model Learner and Base Learners as its sub processes [7]. The training on the process dataset is done in base learner whereas testing part using stacking model learner as shown in Figure 6. Random tree and Neural net are applied as base learners and Naive Bayes (kernel) as stacking model learner. 
Stacking operator delivers model through mod (output) port which is transferred to Apply Model operator through its mod (input) port. The output (out) port of test dataset is connected to Apply Model operator by means of unlabelled (unl) port. The labelled data after applying pre-processed model pass into performance vector in order to appraise the performance of Stacking method.

Confusion matrix generated with class precision, recall and prediction accuracy of Stacking method on test dataset is shown in Table 4.

The stacking method predicted products with warpage, accepted and part does not blow classes as true positives for all cases. Prediction of underweight of part class resulted in 7 cases as true positives, 1 case as false negative (thinning of the weld). Prediction of thinning of the weld class resulted in 8 cases as true positives and 1 case of false positive.

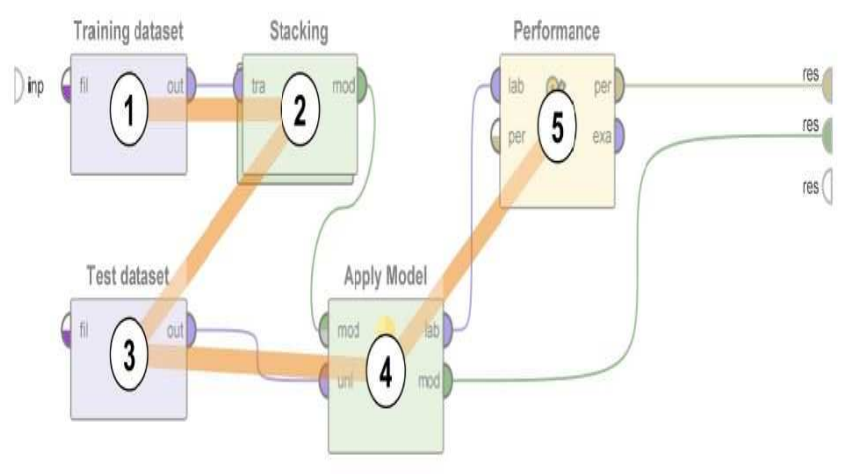

Figure 5: Rapid Miner Process with Stacking Operator.

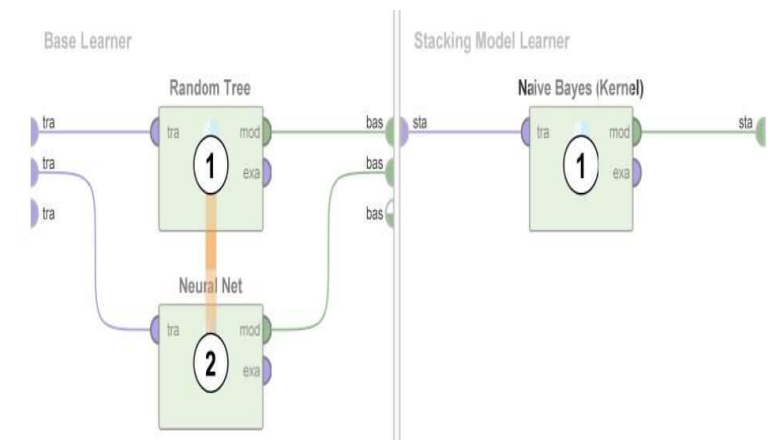

Figure 6:Sub-process of Stacking.

Table 4: Confusion Matrix of Stacking Model

\begin{tabular}{|l|c|c|c|c|c|c|}
\hline & $\begin{array}{c}\text { Accuracy: 97.50 \% } \\
\text { True Under } \\
\text { Weight of Part }\end{array}$ & $\begin{array}{c}\text { True } \\
\text { Warpage }\end{array}$ & $\begin{array}{c}\text { True Thinning } \\
\text { of The Weld }\end{array}$ & $\begin{array}{c}\text { True } \\
\text { Accepted }\end{array}$ & $\begin{array}{c}\text { True Part } \\
\text { Does Not } \\
\text { Blow }\end{array}$ & $\begin{array}{c}\text { Class } \\
\text { Precision }\end{array}$ \\
\hline Pred. Underweight of part & 7 & 0 & 0 & 0 & 0 & $100.00 \%$ \\
\hline Pred. Warpage & 0 & 8 & 0 & 0 & 0 & $100.00 \%$ \\
\hline $\begin{array}{l}\text { Pred. Thinning of the } \\
\text { weld }\end{array}$ & 1 & 0 & 8 & 0 & 0 & $88.89 \%$ \\
\hline Pred. Accepted & 0 & 0 & 0 & 8 & 0 & $100.00 \%$ \\
\hline Pred. Part does not blow & 0 & 0 & 0 & 0 & 8 & $100.00 \%$ \\
\hline Class recall & $87.50 \%$ & $100.00 \%$ & $100.00 \%$ & $100.00 \%$ & $100.00 \%$ & \\
\hline
\end{tabular}

\section{VOTE}

It relies on major vote of inner learners contained in sub-process for classification. The training dataset is given as input to 
vote operator as shown in the process presented in Figure 7. Random Tree, Naive Bayes (Kernel), Neural Net are trained as base learners on training dataset as shown in Figure 8. The vote model depends on major vote of base learners.

Vote operator delivers model through mod (output) port which is transferred to Apply Model operator through its mod (input) port. The test dataset output (out) port is linked to Apply Model operator by means of unlabelled (unl) port. The performance vector is implemented to appraise the performance of Vote method.

Confusion matrix generated with class precision, recall and prediction accuracy of Vote method on test dataset is shown in Table 5.

The vote method predicted products with warpage, accepted and part does not blow classes as true positives for all cases. Prediction of underweight of part class resulted in 7 cases as true positives, 1 case as false negative (thinning of the weld). Prediction of thinning of the weld class resulted in 8 cases as true positives and 1 case as false positive.

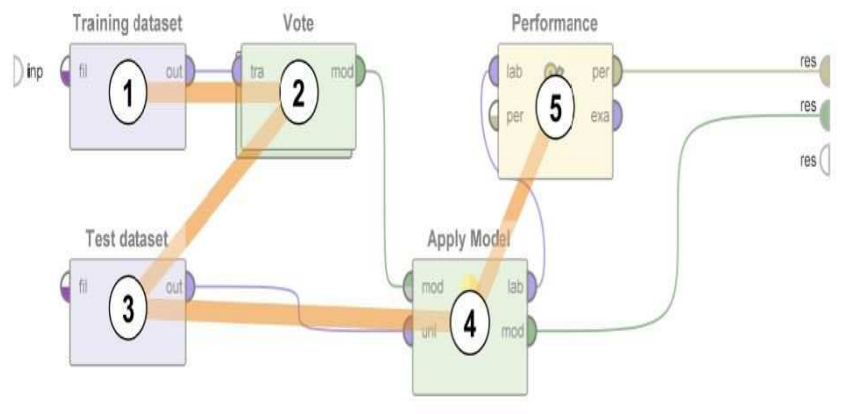

Figure 7: Rapid Miner Process with Vote Operator.

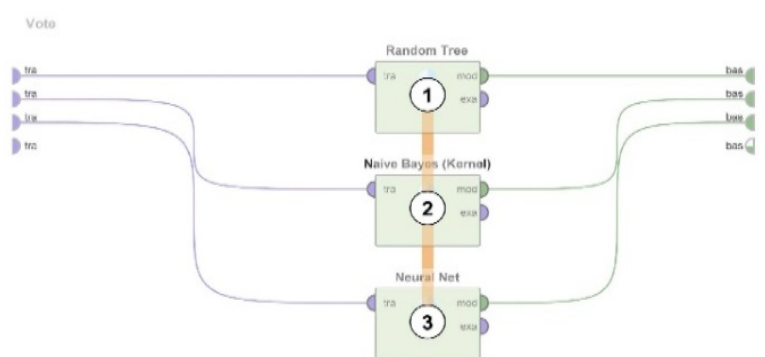

Figure 8: Sub Process of Vote.

Table 5: Confusion Matrix of Vote Model

\begin{tabular}{|l|c|c|c|c|c|c|}
\hline & $\begin{array}{c}\text { True Under } \\
\text { Weight of Part }\end{array}$ & $\begin{array}{c}\text { True } \\
\text { Warpage }\end{array}$ & $\begin{array}{c}\text { True Thinning } \\
\text { of the Weld }\end{array}$ & $\begin{array}{c}\text { True } \\
\text { Accepted }\end{array}$ & $\begin{array}{c}\text { True Part } \\
\text { Does Not Blow }\end{array}$ & $\begin{array}{c}\text { Class } \\
\text { Precision }\end{array}$ \\
\hline Pred. Underweight of part & 7 & 0 & 0 & 0 & 0 & $100.00 \%$ \\
\hline Pred. Warpage & 0 & 8 & 0 & 0 & 0 & $100.00 \%$ \\
\hline Pred. Thinning of the weld & 1 & 0 & 8 & 0 & 0 & $88.89 \%$ \\
\hline Pred. Accepted & 0 & 0 & 0 & 8 & 0 & $100.00 \%$ \\
\hline Pred. Part does not blow & 0 & 0 & 0 & 0 & 8 & $100.00 \%$ \\
\hline Class recall & $87.50 \%$ & $100.00 \%$ & $100.00 \%$ & $100.00 \%$ & $100.00 \%$ & \\
\hline
\end{tabular}

\section{CONCLUSIONS}

Extrusion Blow Molding process dataset is used for building the data mining models by applying Neural Net, Naive Bayes (kernel), Random Tree, Stacking and Vote techniques in RapidMiner. The prediction accuracies of above models are $95 \%$, $90 \%, 82.5 \%, 97.5 \%$ and $97.5 \%$ respectively. Ensemble techniques, stacking and vote models yielded higher prediction 
accuracies among the models. Ensemble models are more dependable in order to arrive near to the optimal process parameter setting in producing defect free products and quality assurance.

\section{REFERENCES}

1. Huang, H-X., and C-M. Liao, 'Prediction of parison swell in plastics extrusion blow molding using a neural network method', Polymer testing, Vol. 21. No. 7, 2002, pp. 745-749.

2. Huang, Geng-Qun, and Han-Xiong Huang, 'Optimizing parison thickness for extrusion blow molding by hybrid method', Journal of Materials Processing Technology., Vol. 182. Nos. 1-3, 2007, pp. 512-518.

3. Huang, Han-Xiong, et al., 'New strategies for predicting parison dimensions in extrusion blow molding', Polymer-Plastics Technology and Engineering., Vol. 50. No. 13, 2011, pp. 1329-1337.

4. Tahboub, Khaldoun K., and Ibrahim A. Rawabdeh, 'A design of experiments approach for optimizing an extrusion blow molding process', Journal of Quality in Maintenance Engineering., Vol. 10. No. 1, 2004, pp. 47-54.

5. Ramana, Dr EV, S. Sapthagiri, and P. Srinivas, 'Data Mining Approach for Quality Prediction of Injection Molding Process Through Statistica SVM, KNN and GC \& RT Techniques', International Journal of Mechanical Engineering and Technology., Vol.7. No. 6, 2016.

6. Ramana, Dr EV, S. Sapthagiri, and P. Srinivas, 'Data Mining Approach for Quality Prediction and Improvement of Injection Molding Process through SANN, GCHAID and Association Rules', International Journal of Mechanical Engineering and Technology., Vol. 7. No. 6, 2016.

7. RapidMiner 8, Operator Reference Manual, RapidMiner GmbH, 2016. 